\title{
THUE INEQUALITIES WITH FEW COEFFICIENTS
}

\author{
PALOMA BENGOECHEA
}

\begin{abstract}
Let $F(x, y)$ be a binary form with integer coefficients, degree $n \geq 3$, and irreducible over the rationals. Suppose that only $s+1$ of the $n+1$ coefficients of $F$ are nonzero. We show that the Thue inequality $|F(x, y)| \leq m$ has $\ll s m^{2 / n}$ solutions provided that the absolute value of the discriminant $D(F)$ of $F$ is large enough. We also give a new upper bound for the number of solutions of $|F(x, y)| \leq m$, with no restriction on the discriminant of $F$ that depends mainly on $s$ and $m$, and slightly on $n$. Our bound becomes independent of $m$ when $m<|D(F)|^{2 /(5(n-1))}$, and also independent of $n$ if $|D(F)|$ is large enough.
\end{abstract}

\section{INTRODUCTION}

Let $F(x, y)$ be a binary form with integer coefficients and degree $n \geq 3$, irreducible over the rationals. Let $m$ be a positive integer. Thue studied in 20] the inequalities

$$
1 \leq|F(x, y)| \leq m
$$

known as Thue inequalities, showing that they have finitely many solutions in integers $x$ and $y$. Mahler [9] showed that Thue inequalities have at most $c(F) m^{2 / n}$ solutions, where $c(F)$ depends only on $F$. In this bound the dependence on $m$ is best possible if $m$ is large. For Thue equations $|F(x, y)|=m$, the dependence on $F$ of $c(F)$ has been progressively replaced by a dependence on the degree $n$, first by Siegel in some special cases, and in general by Evertse [5] in his thesis. Later Bombieri and Schmidt [3] obtained the bound $\ll n^{1+\nu}$ for the number of primitive solutions (solutions $(x, y)$ with $x$ and $y$ coprime), where $\nu$ is the number of prime factors of $m$.

In his fundamental work on diophantine equations $f(x, y)=0$, Siegel 17 conjectured that, when the curve defined by the equation is irreducible and of positive genus, the number of solutions sould be bounded only in terms of the number of nonzero coefficients. In this form, the conjecture is not true; there is no bound independent of $m$ for cubic Thue equations, as the work of Chowla [4, Mahler [10] and Silverman [18] show. However, there have been several subsequent works with the goal of replacing the dependence on the degree by the number of nonzero coefficients. Schmidt was the first in studying this modified version of Siegel's conjecture for Thue equations in

2000 Mathematics Subject Classification. 11D45.

Key words and phrases. Binary Forms, Thue Equations, Thue's inequalities, Fewnomials, sparse forms. 
general, and it turned out to be equally difficult to study Thue inequalities (see his introduction in [15]).

Suppose that $F(x, y)$ has not more than $s+1$ nonzero coefficients, so that

$$
F(x, y)=\sum_{i=0}^{s} a_{i} x^{n_{i}} y^{n-n_{i}}
$$

with $0=n_{0}<n_{1}<\ldots n_{s-1}<n_{s}=n$. Then Schmidt [15] proved that the inequality (11) has

$$
\ll(n s)^{1 / 2} m^{2 / n}\left(1+\log m^{1 / n}\right)
$$

solutions. Here and throughout the paper, the constants implicit in « will be absolute and effectively computable. Thunder [20] could remove the logarithmic factor for many values of $m$. Later, Mueller and Schmidt [14] obtained the second bound

$$
\ll s^{2} m^{2 / n}\left(1+\log m^{1 / n}\right),
$$

hence the number of solutions of (11) is bounded in terms of $s$ and $m$ only. This was proved previously for $s=1$ (i.e. for binomial forms) in [?] and for $s=2$ (i.e. for trinomials) in 13 . Mueller and Schmidt could remove the logarithmic factor in their general bound (4) if $n \geq \max \left(4 s, s \log ^{3} s\right)$. When $n \gg s, F$ is usually called sparse form or fewnomial. They also conjectured that the logarithmic factor should be removed for all forms of degree $n \geq 3$ and, more importantly, that the term $s^{2}$ should be $s$.

Here we establish two new upper bounds for the number of solutions of (11). The bound given in Theorem 1.1 proves Mueller-Schmidt's conjecture for almost all binary forms with given degree.

Theorem 1.1. Let $F(x, y) \in \mathbb{Z}[x, y]$ be an irreducible binary form with $s+1$ nonzero coefficients and degree $n \geq 3$. Assume that the absolute value of the discriminant of $F$ is greater than $(n(n-1))^{8 n(n-1)}$. For each positive integer $m$, the inequality $|F(x, y)| \leq m$ has

$$
\ll s m^{2 / n}
$$

solutions.

Since there are only finitely many $\mathrm{SL}(2, \mathbb{Z})$-equivalence classes of irreducible binary forms of fixed degree and bounded discriminant (see [2]), our result, while stated for a quite strong condition on the discriminant, holds for almost all classes of forms of given degree. (Note that equivalent forms give the same number of solutions to the inequality (11).)

Under a similar condition on the discriminant, an upper bound for the number of solutions to (11) for small values of $m$ and almost all forms is given in [1, following previous works by [6] and [7]. That bound is linear in $s$ when the forms are 'very' sparse, namely when $n \geq s^{2}$.

In Theorem 1.2 we give a new bound that holds for all sparse forms and all integers $m$. It becomes independent of $m$ for small values of $m$ as 
Corollary 1.3 shows, and also independent of $n$ when the absolute value of the discriminant is large enough.

Theorem 1.2. Let $F(x, y) \in \mathbb{Z}[x, y]$ be an irreducible binary form with $s+1$ nonzero coefficients and degree $n \geq 3 s$. Let $D(F)$ and $H(F)$ be the discriminant and the height of $F$ respectively. For each positive integer $m$ the inequality

$$
|F(x, y)| \leq m
$$

has

$$
\ll\left(c(s)\left(1+\log m^{\frac{1}{n}}\right)+\log ^{3} n\right) m^{\frac{2}{n}}|D(F)|^{-\frac{1}{n(n-1)}}
$$

solutions, with

$$
c(s)= \begin{cases}s & \text { if } n \geq s^{4} \\ s \log s & \text { if } 9 s^{2} \leq n<s^{4} \\ s \log s\left(1+\frac{s}{\log H(F)}\right) & \text { if } n<9 s^{2} .\end{cases}
$$

Corollary 1.3. Let $F(x, y) \in \mathbb{Z}[x, y]$ be an irreducible binary form with $s+1$ nonzero coefficients and degree $n \geq 3$ s. Let $D(F)$ be the discriminant of $F$ and $m$ be a positive integer such that

$$
m \leq|D(F)|^{\frac{1}{\left(2+\frac{1}{2}\right)(n-1)}} .
$$

Then $|F(x, y)| \leq m$ has

$$
\ll c(s)+\log ^{3} n
$$

solutions, with $c(s)$ defined by (6).

Moreover, if $|D(F)| \geq(\log n)^{15 n(n-1)}$, then $|F(x, y)| \leq m$ has

$$
\ll c(s)
$$

solutions.

The corollary immediately follows from Theorem 1.2 on noticing that $\log |D(F)|^{\frac{1}{(2+1 / 2) n(n-1)}} \leq|D(F)|^{\frac{1}{2(2+1 / 2) n(n-1)}}$, so when $m$ satisfies (7), we have that

$$
\begin{aligned}
\log m^{1 / n} m^{2 / n} & \leq|D(F)|^{\frac{1 / 4}{\left(1+\frac{1}{4}\right) n(n-1)}}|D(F)|^{\frac{1}{\left(1+\frac{1}{4}\right) n(n-1)}} \\
& \leq|D(F)|^{\frac{1}{n(n-1)}},
\end{aligned}
$$

and when $|D(F)| \geq(\log n)^{15 n(n-1)}$,

$$
m^{2 / n} \log ^{3} n \leq|D(F)|^{\frac{1}{n(n-1)}} .
$$

Note that we will regard $(x, y)$ and $(-x,-y)$ as one solution, and can assume $y \geq 0$ or $x \geq 0$ if convenient. 


\section{Preliminaries}

2.1. Discriminant, Height, and Mahler Measure. For a binary form $G(x, y)$ that factors over $\mathbb{C}$ as

$$
\prod_{i=1}^{n}\left(\alpha_{i} x-\beta_{i} y\right)
$$

the discriminant $D(G)$ of $G$ is given by

$$
D(G)=\prod_{i<j}\left(\alpha_{i} \beta_{j}-\alpha_{j} \beta_{i}\right)^{2} .
$$

Therefore, if we write

$$
G(x, y)=a_{n}\left(x-\gamma_{1} y\right) \ldots\left(x-\gamma_{n} y\right)
$$

we have

$$
D(G)=a_{n}^{2(n-1)} \prod_{i<j}\left(\gamma_{i}-\gamma_{j}\right)^{2} .
$$

The Mahler measure $M(G)$ is defined by

$$
M(G)=\left|a_{n}\right| \prod_{i=1}^{n} \max \left(1,\left|\gamma_{i}\right|\right) .
$$

Mahler [12] showed

$$
M(G) \geq\left(\frac{|D(G)|}{n^{n}}\right)^{\frac{1}{2 n-2}} .
$$

If we write $G(x, y)=a_{n} x^{n}+a_{n-1} x^{n-1} y+\ldots+a_{1} x y^{n-1}+a_{0} y^{n}$, the (naive) height of $G$, denoted by $H(G)$, is defined by

$$
H(G)=\max \left(\left|a_{n}\right|,\left|a_{n-1}\right|, \ldots,\left|a_{0}\right|\right) .
$$

We have

$$
\left(\begin{array}{c}
n \\
\lfloor n / 2\rfloor
\end{array}\right)^{-1} H(G) \leq M(G) \leq(n+1)^{1 / 2} H(G) .
$$

A proof of this fact can be found in [11].

2.2. $G L_{2}(\mathbb{Z})$ Actions and Equivalent Forms. Let $A=\left(\begin{array}{ll}a & b \\ c & d\end{array}\right)$ and define the binary form $F_{A}$ by

$$
F_{A}(x, y)=F(a x+b y, c x+d y) .
$$

Note that

$$
D\left(F_{A}\right)=(\operatorname{det} A)^{n(n-1)} D(F),
$$

and $D\left(F_{A}\right)=D(F)$ if $A \in \mathrm{GL}(2, \mathbb{Z})$.

We say that two binary forms $F$ and $G$ are equivalent if $G= \pm F_{A}$ for some $A \in G L_{2}(\mathbb{Z})$. The number of solutions (and the number of primitive solutions) to Thue inequalities does not change if we replace the binary 
form with an equivalent form. However, $G L_{2}(\mathbb{Z})$-actions do not preserve the number of nonzero coefficients of $F$. Schmidt formulates in [15] a condition that is invariant under $G L_{2}(\mathbb{Z})$ actions. He defines a class $C(t)$ of forms of fixed degree as follows.

Definition of $C(t)$. We define the set $C(t)$ as the set of forms $F(x, y)$ of degree $n$ with integer coefficients, and irreducible over $\mathbb{Q}$, such that for any reals $(u, v) \neq(0,0)$, the form

$$
u F_{x}+v F_{y}
$$

has at most $t$ real zeros.

Note that for $n>0$, the irreducibility of $F$ implies that the form (12) of degree $n-1$ is not identically zero. Note also that for $F \in C(t)$, the derivative $F_{x}(z, 1)$ has $\leq t$ real zeros and $F(z, 1)$ has $\leq t+1$ real zeros. The following is Lemma 2 of 15 .

Lemma 2.1. Suppose $F(x, y)$ is irreducible of degree $n$, and has $s+1$ nonvanishing coefficients. Then $F(x, y) \in C(4 s-2)$.

\section{General strategies for Theorems 1.1 and 1.2}

Definition of Primitive Solutions. A pair $(x, y) \in \mathbb{Z}^{2}$ is called a primitive solution to the inequality (11) if it satisfies the inequality and $\operatorname{gcd}(x, y)=1$.

We note that by this definition the possible solutions $(z, 0)$ and $(0, z)$ are considered primitive if and only if $z= \pm 1$.

Definitions of $N(F, m), P(F, m)$ and $\tilde{P}(F, m)$. For an irreducible binary form $F(x, y) \in \mathbb{Z}[x, y]$ of degree $n \geq 3$, we denote by $N(F, m)$ the number of solutions of $F(x, y) \leq m$ and by $P(F, m)$ the number of primitive solutions. Further, we write $\tilde{P}(F, m)$ for the number of primitive solutions of

$$
2^{-n} m \leq F(x, y)<m .
$$

Note that $\tilde{P}(F, m)$ is not affected if we replace $F$ by an equivalent form. We will show that for $F$ of the form (2),

$$
\tilde{P}(F, m) \ll s m^{2 / n} \quad \text { for }|D(F)|>(n(n-1))^{8 n(n-1)}
$$

and

$$
\tilde{P}(F, m) \ll\left(c(s)\left(1+\log m^{\frac{1}{n}}\right)+\log ^{3} n\right) m^{\frac{2}{n}}|D(F)|^{-\frac{1}{n(n-1)}}
$$

with no restriction on the discriminant $D(F)$.

Once we obtain these upper bounds for $\tilde{P}(F, m)$, it is easy to deduce the same upper bounds for $N(F, m)$. We follow the argument in [15, section 3]. Write

$$
\tilde{P}(F, m)=\left(A_{1}(F)+A_{2}(F)\left(1+\log m^{\frac{1}{n}}\right)\right) m^{\frac{2}{n}},
$$

where $A_{1}(F), A_{2}(F)$ depend only on $F$. 
When $u$ is the integer with $2^{n u} \leq m<2^{n(u+1)}$, then

$$
\begin{aligned}
P(F, m) \leq P\left(F, 2^{n(u+1)}-1\right) & =\sum_{j=1}^{u+1} \tilde{P}\left(F, 2^{n j}\right) \\
& \ll A_{1}(F) \sum_{j=1}^{u+1} 2^{2 j}+A_{2}(F) \sum_{j=1}^{u+1} 2^{2 j}\left(1+\log 2^{j}\right) \\
& \ll A_{1}(F) 2^{2 u}+A_{2}(F) 2^{2 u}(1+u) \\
& \ll\left(A_{1}(F)+A_{2}(F)\left(1+\log m^{\frac{1}{n}}\right)\right) m^{\frac{2}{n}} .
\end{aligned}
$$

Assume (15) and (14). Then (16) implies

$$
P(F, m) \ll\left(c(s)\left(1+\log m^{\frac{1}{n}}\right)+\log ^{3} n\right) m^{\frac{2}{n}}|D(F)|^{-\frac{1}{n(n-1)}}
$$

and

$$
P(F, m) \ll s m^{2 / n} \quad \text { for }|D(F)|>(n(n-1))^{8 n(n-1)} .
$$

Let $\pi(F, m)$ be the number of primitive solutions of $F(x, y)=m$. Then

$$
\pi(F, m)=P(F, m)-P(F, m-1)
$$

(with $P(F, 0)=0$ ). With [.] denoting integer part, we have

$$
\begin{aligned}
N(F, m) & =\sum_{k=1}^{m} \pi(F, k)\left[\left(\frac{m}{k}\right)^{1 / n}\right] \leq m^{1 / n} \sum_{k=1}^{m} \pi(F, k) k^{-1 / n} \\
& =m^{1 / n} \sum_{k=1}^{m}(P(F, k)-P(F, k-1)) k^{-1 / n} \\
& \ll P(F, m) m^{-1 / n} \sum_{k=1}^{m}\left(k^{2 / n}-(k-1)^{2 / n}\right) k^{-1 / n} \\
& \ll P(F, m) m^{-1 / n} \sum_{k=1}^{m} k^{1 / n}-(k-1)^{1 / n} \\
& \ll P(F, m)
\end{aligned}
$$

since the sum is telescoping.

Hence the whole difficulty in Theorems 1.1 and 1.2 is to bound $\tilde{P}(F, m)$, the number of solutions to (13). We will split the count of possible solutions to (13) into small and large solutions for Theorem 1.1, and small, medium and large solutions for Theorem 1.2. The definitions of small and large will differ for the two theorems. However, a common argument is used for small solutions. We use the classical decomposition of $F(x, y)$ into linear forms introduced by Bombieri and Schmidt in [3] and used in several works afterwards to estimate small solutions. We will also use a lemma by Mueller and Schmidt (recorded here as Lemma 4.6) in a similar way as in [1, section 4]. This lemma is crucial for the treatment of large solutions in Theorem 1.1. Mueller and Schmidt formulated their lemma in terms of forms with $s$ 
nonzero coefficients, but in fact this lemma can be applied to a larger class of forms, and this is the reason why we exploit it so much in this paper. We combine it with an argument from [20, which is based on the LewisMahler inequality (Lemma 7.1) on the approximation by the roots of $F$ to the rationals $\frac{x}{y}$, where $(x, y)$ are integral solutions to (1), together with a gap type result due to Schmidt [16].

The bound for medium solutions for Theorem 1.2 is an extension of the argument in [1, section 5]. The bound for large solutions is given by a result of Mueller and Schmidt in 14. In the calculation of the three bounds (for small, medium and large solutions) for Theorem 1.2, we use some results that need the assumption that $F$ has $s$ nonzero coefficients, and some other results that need the assumption that $F$ has minimal Mahler measure. Combining both assumptions can be a problem a priori, and this may be a reason for the existence of the two simultaneous papers [15] and [14, where each of them assumes exactly one of the two hypothesis. The way we are able to combine both hypothesis here (in section 8) is also new.

The rest of the manuscript is organized as follows. We discuss the argument for small solutions for both theorems in section 4, and then apply it to the specific definitions of 'small' in sections 6 and 9. In sections 5-7 we focus on Theorem 1.1. In section 5 we give the definitions of small and large solutions, we give the results that essentially count them (see Propositions 5.1 and 5.2$)$ and bound $\tilde{P}(F, m)$ for large discriminants assuming them. We prove Propositions 5.1 and 5.2 in sections 6 and 7 respectively.

In sections 8-10 we focus on Theorem 1.2. In section 8 we give the definitions of small, medium and large solutions, and again we give the results that essentially count those solutions (see Propositions 8.3, 8.2 and 8.1), and bound $\tilde{P}(F, m)$ assuming them. In sections 9 and 10 we prove Propositions 8.3 and 8.2 respectively.

\section{SMall SOlutions}

Let $F(x, y) \in \mathbb{Z}[x, y]$ be an irreducible binary form of degree $n \geq 3$ that lies in $C(4 s-2)$. Let $M$ be the smallest Mahler measure among the forms equivalent to $F$, and $m$ be a positive integer such that

$$
m \leq \frac{M}{100^{n}} .
$$

Under these assumptions, we give an upper bound for the number of solutions $(x, y)$ of (13) that satisfy $1 \leq y<Y$, for a constant $Y$. Our bound will of course depend on $Y$ and will be applied later to two different values of $Y$. Similarly we give an upper bound for the number of solutions with $1 \leq x<Y$.

We suppose that there is at least one primitive solution of (13) with $1 \leq y<Y$. We fix such a solution $\left(x^{*}, y^{*}\right)$ such that $y^{*} \leq y$ for all primitive solutions $(x, y)$. Note that any primitive solution $(x, y) \neq( \pm 1,0)$ will have 
$y \geq 1$.

Definition of $L_{i}(x, y)$. For the binary form

$$
F(x, y)=a_{n}\left(x-\alpha_{1} y\right) \ldots\left(x-\alpha_{n} y\right),
$$

we define

$$
L_{i}(x, y)=x-\alpha_{i} y \quad(i=1, \ldots, n) .
$$

Here $\alpha_{1}, \ldots, \alpha_{n}$ are the roots of the polynomial $F(x, 1)$.

Given $\boldsymbol{x}=(x, y)$ and $\boldsymbol{x}^{\prime}=\left(x^{\prime}, y^{\prime}\right)$, we define

$$
\mathcal{D}\left(\boldsymbol{x}, \boldsymbol{x}^{\prime}\right)=x y^{\prime}-x^{\prime} y .
$$

Lemma 4.1. Suppose $\boldsymbol{x}=(x, y)$ is a primitive solution of (13). We have

$$
\frac{L_{i}\left(x^{*}, y^{*}\right)}{L_{i}(x, y)}-\frac{L_{j}\left(x^{*}, y^{*}\right)}{L_{j}(x, y)}=\left(\beta_{j}-\beta_{i}\right) \mathcal{D}\left(\boldsymbol{x}, \boldsymbol{x}^{*}\right),
$$

where $\beta_{1}, \ldots, \beta_{n}$ depend on $(x, y)$ and are such that the form

$$
J(u, w)=F(x, y)\left(u-\beta_{1} w\right) \ldots\left(u-\beta_{n} w\right)
$$

is equivalent to $F$.

Proof. This is Lemma 3 of [3].

For a primitive solution $(x, y)$ of (13), we have

$$
\frac{F\left(x^{*}, y^{*}\right)}{F(x, y)}<2^{n} \text {. }
$$

Let $i_{0} \in\{1, \ldots, n\}$ be the index such that

$$
\left|\frac{L_{i_{0}}\left(x^{*}, y^{*}\right)}{L_{i_{0}}(x, y)}\right|=\min _{1 \leq i \leq n}\left|\frac{L_{i}\left(x^{*}, y^{*}\right)}{L_{i}(x, y)}\right|,
$$

so that, by (21),

$$
\left|\frac{L_{i_{0}}\left(x^{*}, y^{*}\right)}{L_{i_{0}}(x, y)}\right| \leq 2
$$

By Lemma 4.1 and (23),

$$
\frac{\left|L_{i}\left(x^{*}, y^{*}\right)\right|}{\left|L_{i}(x, y)\right|} \geq\left|\beta_{i_{0}}-\beta_{i}\right|\left|\mathcal{D}\left(\boldsymbol{x}, \boldsymbol{x}^{*}\right)\right|-2 .
$$

For the complex conjugate $\bar{\beta}_{i_{0}}$ of $\beta_{i_{0}}$, we also have

$$
\frac{\left|L_{i}\left(x^{*}, y^{*}\right)\right|}{\left|L_{i}(x, y)\right|} \geq\left|\bar{\beta}_{i_{0}}-\beta_{i}\right|\left|\mathcal{D}\left(\boldsymbol{x}, \boldsymbol{x}^{*}\right)\right|-2 .
$$

Hence

$$
\frac{\left|L_{i}\left(x^{*}, y^{*}\right)\right|}{\left|L_{i}(x, y)\right|} \geq\left|\operatorname{Re}\left(\beta_{i_{0}}\right)-\beta_{i}\right|\left|\mathcal{D}\left(\boldsymbol{x}, \boldsymbol{x}^{*}\right)\right|-2,
$$


where $\operatorname{Re}\left(\beta_{i_{0}}\right)$ is the real part of $\beta_{i_{0}}$. Now we choose an integer $d=d(x, y)$, with $\left|\operatorname{Re}\left(\beta_{i_{0}}\right)-d\right| \leq 1 / 2$, and we obtain

$$
\frac{\left|L_{i}\left(x^{*}, y^{*}\right)\right|}{\left|L_{i}(x, y)\right|} \geq\left(\left|d-\beta_{i}\right|-\frac{1}{2}\right)\left|\mathcal{D}\left(\boldsymbol{x}, \boldsymbol{x}^{*}\right)\right|-2,
$$

for $i=1, \ldots, n$.

Definition of the sets $\mathfrak{X}_{i}$. Let $\mathfrak{X}_{i}$ be the set of primitive solutions $(x, y) \neq$ $\left(x^{*}, y^{*}\right)$ of (13) with $1 \leq y \leq Y$ and $\left|L_{i}(x, y)\right| \leq \frac{1}{2 y}$, where $1 \leq i \leq n$.

We note that if $\alpha_{i}$ and $\alpha_{j}$ are complex conjugates then $\mathfrak{X}_{i}=\mathfrak{X}_{j}$.

Lemma 4.2. Suppose $(x, y)$ and $\left(x^{\prime}, y^{\prime}\right)$ are two distinct primitive solutions in $\mathfrak{X}_{i}$, with $1 \leq y \leq y^{\prime}$. Then

$$
\frac{y^{\prime}}{y} \geq \frac{2}{11} \max \left(1,\left|\beta_{i}(x, y)-d(x, y)\right|\right) .
$$

Proof. We follow the proof of Lemma 4 of [3] and Lemma 4.3 of [1]. We have that

$$
\begin{aligned}
1 \leq\left|y^{\prime} x-y x^{\prime}\right| & \leq y\left|L_{i}\left(x^{\prime}, y^{\prime}\right)\right|+y^{\prime}\left|L_{i}(x, y)\right| \\
& \leq \frac{y}{2 y^{\prime}}+y^{\prime}\left|L_{i}(x, y)\right| \\
& \leq \frac{1}{2}+y^{\prime}\left|L_{i}(x, y)\right|
\end{aligned}
$$

Therefore,

$$
y^{\prime} \geq \frac{1}{2\left|L_{i}(x, y)\right|} .
$$

Combining this with (25), we get

$$
\begin{aligned}
\frac{y^{\prime}}{y} & \geq \frac{1}{2}\left(\left|d(x, y)-\beta_{i}(x, y)\right|-\frac{1}{2}\right) \frac{\left|\mathcal{D}\left(\boldsymbol{x}, \boldsymbol{x}^{*}\right)\right|}{y\left|L_{i}\left(x^{*}, y^{*}\right)\right|}-\frac{1}{y\left|L_{i}\left(x^{*}, y^{*}\right)\right|} \\
& \geq \frac{\left(\left|d-\beta_{i}\right|-\frac{5}{2}\right)\left|\mathcal{D}\left(\boldsymbol{x}, \boldsymbol{x}^{*}\right)\right|}{2 y\left|L_{i}\left(x^{*}, y^{*}\right)\right|} .
\end{aligned}
$$

Now,

$$
\begin{aligned}
\frac{\left|\mathcal{D}\left(\boldsymbol{x}, \boldsymbol{x}^{*}\right)\right|}{y\left|L_{i}\left(x^{*}, y^{*}\right)\right|} & =\frac{\left|\frac{x}{y}-\frac{x^{*}}{y^{*}}\right|}{\left|\frac{x^{*}}{y^{*}}-\alpha_{i}\right|} \geq \frac{\left|\frac{x}{y}-\frac{x^{*}}{y^{*}}\right|}{\left|\frac{x^{*}}{y^{*}}-\frac{x}{y}\right|+\frac{1}{2 y^{2}}} \\
& \geq \frac{1}{1+\frac{1}{2 y^{2}\left|\frac{x^{*}}{y^{*}}-\frac{x}{y}\right|}} \geq \frac{2}{3}
\end{aligned}
$$


where the last inequality is because $\left|\frac{x^{*}}{y^{*}}-\frac{x}{y}\right| \geq \frac{1}{y^{*} y}$ and so

$$
\frac{1}{2 y^{2}\left|\frac{x^{*}}{y^{*}}-\frac{x}{y}\right|} \leq \frac{y^{*}}{2 y} \leq \frac{1}{2} \text {. }
$$

Therefore, by (27) and (28), we have

$$
\begin{aligned}
\frac{y^{\prime}}{y} & \geq \max \left(1,\left(\left|d-\beta_{i}\right|-\frac{5}{2}\right) \frac{1}{3}\right) \\
& \geq \frac{3}{11} \max \left(1, \frac{2}{3}\left|d-\beta_{i}\right|\right),
\end{aligned}
$$

where in the second inequality we used that $\max \left(1, \frac{\zeta}{2}-a\right) \geq \frac{1}{2 a+2} \max (1, \zeta)$ with $\zeta=\frac{2}{3}\left|d-\beta_{i}\right|$ and $a=\frac{5}{6}$.

Lemma 4.3. Suppose $(x, y) \notin \mathfrak{X}_{i} \cup\left\{\left(x^{*}, y^{*}\right)\right\}$ is a primitive solution of (13) with $y>0$. Then

$$
\left|d(x, y)-\beta_{i}(x, y)\right| \leq \frac{11}{2} .
$$

Proof. By (25), we have

$$
\left|d-\beta_{i}\right| \leq\left(\frac{\left|L_{i}\left(x^{*}, y^{*}\right)\right|}{\left|L_{i}(x, y)\right|}+2\right) \frac{1}{\left|\mathcal{D}\left(\boldsymbol{x}, \boldsymbol{x}^{*}\right)\right|}+\frac{1}{2} .
$$

Since $\left|\frac{x}{y}-\frac{x^{*}}{y^{*}}\right| \geq \frac{1}{y y^{*}}$ and we are assuming $\left|L_{i}(x, y)\right|>\frac{1}{2 y}$, we have $\frac{\left|L_{i}\left(x^{*}, y^{*}\right)\right|}{\left|L_{i}(x, y)\right|\left|\mathcal{D}\left(\boldsymbol{x}, \boldsymbol{x}^{*}\right)\right|} \leq \frac{\left|\alpha_{i}-\frac{x}{y}\right|+\left|\frac{x}{y}-\frac{x^{*}}{y^{*}}\right|}{y^{2}\left|\alpha_{i}-\frac{x}{y}\right|\left|\frac{x}{y}-\frac{x^{*}}{y^{*}}\right|} \leq \frac{1}{y^{2}\left|\frac{x}{y}-\frac{x^{*}}{y^{*}}\right|}+\frac{1}{y^{2}\left|\alpha_{i}-\frac{x}{y}\right|} \leq 3$.

Therefore, using that $\left|\mathcal{D}\left(\boldsymbol{x}, \boldsymbol{x}^{*}\right)\right| \geq 1$, we conclude that

$$
\left|d-\beta_{i}\right| \leq \frac{11}{2} \text {. }
$$

Let $(x, y)$ be a fixed primitive solution to (13). Recall that the form

$$
J(u, w)=F(x, y)\left(u-\beta_{1} w\right) \ldots\left(u-\beta_{n} w\right)
$$

is equivalent to $F$ (see Lemma 4.1). Hence the form

$$
\hat{J}(u, w)=F(x, y)\left(u-\left(\beta_{1}-d\right) w\right) \ldots\left(u-\left(\beta_{n}-d\right) w\right)
$$

is also equivalent to $F$. Therefore,

$$
\prod_{i=1}^{n} \max \left(1,\left|\beta_{i}(x, y)-d(x, y)\right|\right) \geq \frac{M(\hat{J})}{F(x, y)} \geq \frac{M}{m} \text {. }
$$


Definition of $\mathfrak{X}$. For each set $\mathfrak{X}_{i}(i=1, \ldots, n)$ that is not empty, let $\left(x^{(i)}, y^{(i)}\right) \in \mathfrak{X}_{i}$ be the element with the largest value of $y$. Consider the set of primitive solutions of (13) that are not $\left(x^{*}, y^{*}\right)$ and with $1 \leq y \leq Y$ minus the elements $\left(x^{(1)}, y^{(1)}\right), \ldots,\left(x^{(n)}, y^{(n)}\right)$. We define $\mathfrak{X}$ to be that set together with the elements $\left(x^{(i)}, y^{(i)}\right) \in \mathfrak{X}_{i} \cap \mathfrak{X}_{j}(i, j \in\{1, \ldots, n\})$ such that $\left(x^{(i)}, y^{(i)}\right) \neq\left(x^{(j)}, y^{(j)}\right)$.

The rest of this section is devoted to bound the cardinal of

$$
\mathfrak{X} \cup\left\{\left(x^{(i)}, y^{(i)}\right)\right\}_{1 \leq i \leq n} .
$$

Lemma 4.4. For any fixed $i \in\{1, \ldots, n\}$, we have

$$
\prod_{(x, y) \in \mathfrak{X}} \frac{2}{11} \max \left(1,\left|\beta_{i}(x, y)-d(x, y)\right|\right) \leq Y .
$$

Proof. Fix $i \in\{1, \ldots, n\}$. Suppose that the set $\mathfrak{X}_{i}$ is not empty. We index the elements of $\mathfrak{X}_{i}$ as

$$
\left(x_{1}^{(i)}, y_{1}^{(i)}\right), \ldots,\left(x_{v}^{(i)}, y_{v}^{(i)}\right)
$$

so that $y_{1}^{(i)} \leq \ldots \leq y_{v}^{(i)}$ (note that $\left.\left(x_{v}^{(i)}, y_{v}^{(i)}\right)=\left(x^{(i)}, y^{(i)}\right)\right)$. By Lemma 4.2,

$$
\frac{2}{11} \max \left(1,\left|\beta_{i}\left(x_{k}^{(i)}, y_{k}^{(i)}\right)-d\left(x_{k}^{(i)}, y_{k}^{(i)}\right)\right|\right) \leq \frac{y_{k+1}^{(i)}}{y_{k}^{(i)}}
$$

for $k=1, \ldots, v-1$, so we have

$$
\prod_{(x, y) \in \mathfrak{X} \cap \mathfrak{X}_{\mathfrak{i}}} \frac{2}{11} \max \left(1,\left|\beta_{i}(x, y)-d(x, y)\right|\right) \leq Y .
$$

For any solution $(x, y) \in \mathfrak{X}$ that does not belong to $\mathfrak{X}_{\mathfrak{i}}$, by Lemma 4.3 , we have

$$
\frac{2}{11} \max \left(1,\left|\beta_{i}(x, y)-d(x, y)\right|\right) \leq 1 .
$$

This, together with (32), completes the proof of the lemma.

Next we will establish inequalities similar to (30) for the solutions $\left(x^{(i)}, y^{(i)}\right)$ which do not belong to $\mathfrak{X}$. Lemma 4.6 will be crucial in order to find a clever way of counting those solutions in terms of $s$. Lemma 4.6 is in fact Lemma 7 of [14, although in [14 the lemma is stated for polynomials with few nonzero coefficients, whereas we state it for any polynomial that belongs to $C(4 s-2)$. The proof only involves the fact that the polynomials and their derivatives have few real zeros; we reproduce it here for convenience of the reader. Put

$$
R=n^{800 \log ^{2} n}
$$

We first need the following result. 
Lemma 4.5. Let $f(z)$ be a polynomial of degree $n$ with real coefficients. Suppose that $f(x) f^{\prime}(x) \neq 0$ for real $x \in I$, where $I$ is an interval $X_{1}<$ $x<X_{2}$, or a half line $x<X_{2}$, or $x>X_{1}$. Suppose there are $u>1$ roots $\gamma_{j}=x_{j}+i y_{j}(j=1, \ldots, u)$ with real parts $x_{j} \in I$. Then there is a root $\gamma_{\ell}$ among these $u$ roots such that for every real $\zeta$,

$$
\left|\zeta-\gamma_{\ell}\right|<R \min _{1 \leq i \leq u}\left|\zeta-\gamma_{i}\right|
$$

Proof. This is Lemma 6 of [14].

Lemma 4.6. There is a set $\boldsymbol{S}$ of roots $\alpha_{i}$ of $F(x, 1)$ with $|\boldsymbol{S}| \leq 12$ s-3 such that for any real $\zeta$,

$$
\min _{\alpha_{\ell} \in S}\left|\zeta-\alpha_{\ell}\right| \leq R \min _{1 \leq i \leq n}\left|\zeta-\alpha_{i}\right| .
$$

Proof. Let $u=4 s-2$ and $f(x)=F(x, 1)$. Since $F(x, y) \in C(u), f(x)$ has $\leq u+1$ real zeros and its derivative $f^{\prime}(x)$ has $\leq u$ real zeros, so that $f(x) f^{\prime}(x)$ has $\leq 2 u+1$ real zeros. Thus the real numbers $x$ with $f(x) f^{\prime}(x) \neq 0$ fall into $\leq 2 u+2$ intervals (or half lines) $I$. Let $\boldsymbol{S}$ consist on the one hand of the real zeros of $f(x)$, and on the other hand, for each interval $I$ as above for which there are roots of $f(x)$ with real part in $I$, pick a $\gamma_{\ell}$ according to Lemma 4.5. The set $\boldsymbol{S}$ so attained will have $|\boldsymbol{S}| \leq u+1+2 u+2=3 u+3=12 s-3$.

Let $\boldsymbol{S}=\left\{\alpha_{1}, \ldots, \alpha_{t}\right\}$, with $t \leq 12 s-3$.

Definition of the set $\mathfrak{X}_{0}$. Let

$$
\mathfrak{X}_{0}=\left\{\left(x^{(i)}, y^{(i)}\right) \in \mathfrak{X}_{i} \backslash\left\{\mathfrak{X}_{1} \cup \ldots \cup \mathfrak{X}_{t} \cup \mathfrak{X}\right\}\right\}_{t<i \leq n} .
$$

Let $\left(x^{(i)}, y^{(i)}\right) \in \mathfrak{X}_{0}$. By Lemma 4.6 there exists $\ell \in\{1, \ldots, t\}$ such that

$$
\left|L_{i}\left(x^{(i)}, y^{(i)}\right)\right| \geq \frac{\left|L_{\ell}\left(x^{(i)}, y^{(i)}\right)\right|}{R} \geq \frac{1}{2 y^{(i)} R},
$$

where the last inequality is because $\left(x^{(i)}, y^{(i)}\right) \notin \mathfrak{X}_{\ell}$. Combining this with (25), we obtain

$$
\left|d\left(x^{(i)}, y^{(i)}\right)-\beta_{i}\left(x^{(i)}, y^{(i)}\right)\right| \leq \frac{2\left|L_{i}\left(x^{*}, y^{*}\right)\right| y^{(i)} R+2}{\left|\mathcal{D}\left(\boldsymbol{x}^{(i)}, \boldsymbol{x}^{*}\right)\right|}+\frac{1}{2} .
$$

Using (28), we obtain

$$
\max \left(1,\left|d\left(x^{(i)}, y^{(i)}\right)-\beta_{i}\left(x^{(i)}, y^{(i)}\right)\right|\right) \leq 3 R+\frac{5}{2} .
$$

Note that a solution $\left(x^{(j)}, y^{(j)}\right)$ belongs to $\mathfrak{X}_{i}$ if and only if $\left(x^{(j)}, y^{(j)}\right)=$ $\left(x^{(i)}, y^{(i)}\right)$ or $\left(x^{(j)}, y^{(j)}\right) \in \mathfrak{X}$, and in this case $\left(x^{(j)}, y^{(j)}\right) \notin \mathfrak{X}_{0}$. Hence, by (35) and Lemma 4.3,

$$
\prod_{(x, y) \in \mathfrak{X}_{0}} \frac{2}{11} \max \left(1,\left|d(x, y)-\beta_{i}(x, y)\right|\right) \leq \frac{6 R+5}{11}
$$


for $i=1, \ldots, n$. Taking the product of (301) and (36) for $i=1, \ldots, n$ we find

$$
\left(\frac{M}{\left(\frac{11}{2}\right)^{n} m}\right)^{\left|\mathfrak{X} \cup \mathfrak{X}_{0}\right|} \leq\left(Y \frac{6 R+5}{11}\right)^{n} \text {. }
$$

Therefore,

$$
\left|\mathfrak{X} \cup \mathfrak{X}_{0}\right|<\frac{n \log Y+n \log (6 R+5)}{\log \left(M /\left(6^{n} m\right)\right)} .
$$

The primitive solutions $(x, y)$ of (13) with $1 \leq y \leq Y$ are in

$$
\mathfrak{X} \cup \mathfrak{X}_{0} \cup\left\{\left(x^{(i)}, y^{(i)}\right)\right\}_{1 \leq i \leq t} \cup\left\{\left(x^{*}, y^{*}\right)\right\},
$$

so there are $\ll\left|\mathfrak{X} \cup \mathfrak{X}_{0}\right|+s$ of them.

The number of primitive solutions of (13) with $1 \leq x \leq Y$ can be estimated in a similar way, by considering the form

$$
F(x, y)=a_{0}\left(y-\gamma_{1} x\right) \cdot \ldots \cdot\left(y-\gamma_{n} x\right)
$$

and putting $L_{i}(x, y)=y-\gamma_{i} x$. Here $\gamma_{1}, \ldots, \gamma_{n}$ are the roots of the polynomial $F(1, y)$.

\section{Bound FOr $\tilde{P}(F, m)$ FOR LARGE DISCRIMINANTS}

Let $F(x, y) \in \mathbb{Z}[x, y]$ be an irreducible binary form of degree $n \geq 3$ that lies in $C(4 s-2)$ such that

$$
|D(F)|>(n(n-1))^{8 n(n-1)} .
$$

Further, assume that $F$ has the smallest Mahler measure among all its equivalent forms. We will say for abreviation that $F$ has minimal Mahler measure. Later we need this assumption in order to use simultaneously section 4 and Lemma 7.1. In Lemma 7.1, the Mahler measure of $F$ is involved, whereas in section 4 we work with the smallest Mahler measure among all forms equivalent to $F$. We need both measures to be the same. Let $M=M(F)$ be the minimal Mahler measure. We first count the number of solutions to (13) such that

$$
m \leq \frac{|D(F)|^{\frac{1}{2(n-1)}}}{e^{200 n}} .
$$

Note that, if $m$ satisfies (39), then by (8) we also have

$$
m \leq \frac{M}{e^{200 n}} \text {. }
$$

Let

$$
Y_{0}=(M / m)^{5} \text {. }
$$

Relative to the quantity $Y_{0}$, we call a solution $(x, y) \in \mathbb{Z}^{2}$

$$
\begin{array}{lll}
\text { small if } & 0 \leq y \leq Y_{0}, \\
\text { large } & \text { if } & y>Y_{0} .
\end{array}
$$


Proposition 5.1. Let $F \in C(4 s-2)$ and $m$ be a positive integer satisfying (39). Assume (38) and that $F$ has minimal Mahler measure. The inequality (13) has $\ll n$ small solutions.

Proposition 5.2. Let $F \in C(4 s-2)$ and $m$ be a positive integer satisfying (39). Assume (38) and that $F$ has minimal Mahler measure. The inequality (13) has $\ll s \log \log m^{1 / n}$ large solutions.

Hence the number of solutions of (13) with $F$ having minimal Mahler measure, with (38), and $m$ satisfying (39) is

$$
\ll n+s \log \log m^{1 / n} .
$$

Now we use the argument in [3, section II] to derive an upper bound for $\tilde{P}(F, m)$ for any positive integer $m$ and with no need that $F$ has minimal Mahler measure. Pick the smallest prime $p$ that satisfies

$$
p \geq e^{400} m^{\frac{2}{n}}|D(F)|^{-\frac{1}{n(n-1)}} .
$$

Note that

$$
p<2 e^{400} m^{\frac{2}{n}}|D(F)|^{-\frac{1}{n(n-1)}} .
$$

Let

$$
A_{0}=\left(\begin{array}{ll}
1 & 0 \\
0 & p
\end{array}\right), \quad A_{j}=\left(\begin{array}{cc}
p & j \\
0 & 1
\end{array}\right) \quad(j=1, \ldots, p) .
$$

We have that $F_{A_{j}} \in C(4 s-2)$ for $j=0, \ldots, p$. We also have $\mathbb{Z}^{2}=\cup_{j=0}^{p} A_{j} \mathbb{Z}^{2}$, so that

$$
\tilde{P}(F, m) \leq \sum_{j=0}^{p} \tilde{P}\left(F_{A_{j}}, m\right) .
$$

Let $F_{A_{j}}^{\prime}$ be a form equivalent to $F_{A_{j}}$ that has minimal Mahler measure.

For $j=0, \ldots, p$, by (11) and (43),

$$
\left|D\left(F_{A_{j}}^{\prime}\right)\right|=p^{n(n-1)}|D(F)| \geq e^{400 n(n-1)} m^{2 n-2},
$$

so $m \leq\left|D\left(F_{A_{j}}^{\prime}\right)\right|^{\frac{1}{2(n-1)}} / e^{200 n}$, and $\left|D\left(F_{A_{j}}^{\prime}\right)\right|$ satisfies (38), so we can apply Propositions 5.1 and 5.2 to $F_{A_{j}}^{\prime}$. Hence

$$
\tilde{P}\left(F_{A_{j}}, m\right)=\tilde{P}\left(F_{A_{j}}^{\prime}, m\right) \ll n+s \log \log m^{1 / n} .
$$

By (45) and (44), we obtain that

$$
\begin{aligned}
\tilde{P}(F, m) & \ll(p+1)\left(n+s \log \log m^{\frac{1}{n}}\right) \\
& \ll\left(n+s \log \log m^{\frac{1}{n}}\right) m^{\frac{2}{n}}|D(F)|^{-\frac{1}{n(n-1)}} .
\end{aligned}
$$

If $|D(F)|^{\frac{1}{n(n-1)}}>\log \log m^{\frac{1}{n}}$, (14) follows from (46) and the assumption (38). 
If $|D(F)|^{\frac{1}{n(n-1)}} \leq \log \log m^{\frac{1}{n}}$, then by (38) we also have that $\log \log m \geq$ $(n(n-1))^{8}$, and the result below, which is part of the Corollary of Theorem 2 in [20] (with $\varepsilon=1 / 2$ ) concludes the proof of (14).

Proposition 5.3. Let $F(x, y) \in \mathbb{Z}[x, y]$ be an irreducible binary form with $s+1$ nonzero coefficients and degree $n \geq 3$. Let $m$ be a positive integer. If $|D(F)|^{1 / n(n-1)} \leq \log \log m$ and $\log \log m \geq(n(n-1))^{8}$, then $|F(x, y)| \leq m$ has $\ll m^{2 / n}$ solutions.

\section{Proof of Proposition 5.1}

We apply (37) to $Y=Y_{0}$ defined by (41). We have, by (39) and (34),

$$
\begin{aligned}
n \log Y_{0}+n \log (6 R+5) & \ll n\left(\log \frac{M}{6^{n} m}+\log 6^{n}\right)+n \log ^{3} n \\
& \ll n \log \frac{M}{6^{n} m}+n^{2}
\end{aligned}
$$

and

$$
\log \left(M /\left(6^{n} m\right)\right) \gg n .
$$

Hence $\left|\mathfrak{X} \cup \mathfrak{X}_{0}\right| \ll n$ and we conclude Proposition 5.1,

\section{Large solutions, Proof of Proposition 5.2}

Let $F(x, y)=\sum_{i=0}^{n} a_{i} x^{i} y^{n-i} \in \mathbb{Z}[x, y]$ be an irreducible binary form that lies in $C(4 s-2)$ and satisfies (38). Let $m$ be a positive integer that satisfies (39).

The following lemma is a version of the Lewis-Mahler inequality [8], refined by Bombieri and Schmidt [3. Lemma 4] and later written by Stewart [19. Lemma 3] in terms of the discriminant of $F$ instead of the height.

Lemma 7.1. For every pair of integers $(x, y)$ with $y \neq 0$,

$$
\min _{1 \leq i \leq n}\left|\alpha_{i}-\frac{x}{y}\right| \leq \frac{2^{n-1} n^{(n-1) / 2} M(F)^{n-2}|F(x, y)|}{|D(F)|^{1 / 2} y^{n}} .
$$

Let $(x, y)$ be a solution to (13) with $y>Y_{0}$, and let

$$
\left|\alpha_{j}-\frac{x}{y}\right|=\min _{1 \leq i \leq t}\left|\alpha_{i}-\frac{x}{y}\right|
$$

where $t$ is the cardinal of the set $\boldsymbol{S}$ defined by Lemma 4.6. By Lemmas 7.1 and 4.6 ,

$$
\left|\alpha_{j}-\frac{x}{y}\right| \leq \frac{R 2^{n-1} n^{(n-1) / 2} M(F)^{n-2} m}{|D(F)|^{1 / 2} y^{n}}
$$


with $R$ defined in (34). On noticing that $e^{200 n(n-1)}>R 2^{n} n^{(n-1) / 2}$ and using (39), we have

$$
\begin{aligned}
\left|\alpha_{j}-\frac{x}{y}\right| & <\frac{(M(F) / m)^{n-2}}{2 y^{n}} \\
& \leq \frac{1}{2 y^{n-(n-2) / 5}},
\end{aligned}
$$

where in the last inequality we used (41) and $y>Y_{0}$. Let $\left(x_{1}, y_{1}\right),\left(x_{2}, y_{2}\right), \ldots$ be the primitive solutions to (13) with $y>Y_{0}$ and ordered so that

$$
Y_{0}<y_{1} \leq y_{2} \leq \ldots
$$

For all $i>1$, by (48) we have

$$
\frac{1}{y_{i} y_{i-1}} \leq\left|\frac{x_{i}}{y_{i}}-\frac{x_{i-1}}{y_{i-1}}\right|<\frac{1}{y_{i-1}^{n-(n-2) / 5}} .
$$

Thus

$$
y_{i}>y_{i-1}^{n-1-(n-2) / 5}=y_{i-1}^{(4 n-3) / 5}>(M / m)^{4 n-3} .
$$

On noting that $n-3 \sqrt{n} / 2>3 / 10$ for $n \geq 3$ and $\frac{3}{10}(4 n-3)>n-2$, by (49) we have

$$
y_{i}^{n-3 \sqrt{n} / 2}>y_{i}^{3 / 10}>(M(F) / m)^{n-2} .
$$

Hence, by (47),

$$
\left|\alpha_{j}-x_{i} / y_{i}\right|<y_{i}^{-3 \sqrt{n} / 2}
$$

for all $i>1$. By [16, Theorem 9A, Chapter 2], the number of solutions to (50) is

$$
\ll 1+\log \log h\left(\alpha_{j}\right) / \log n,
$$

where $h\left(\alpha_{j}\right)$ is the absolute height of $\alpha_{j}$ defined in [16, $\S 7$ Chapter 1]. By [16. Lemma 2A Chapter 3],

$$
h\left(\alpha_{j}\right)=\operatorname{cont}(F)^{-1}\left(\left|a_{n}\right| \prod_{k=1}^{n} \sqrt{1+\left|\alpha_{k}\right|^{2}}\right)^{1 / n} .
$$

Here $\operatorname{cont}(F)=\operatorname{gcd}\left(\left|a_{0}\right|, \ldots,\left|a_{n}\right|\right)$. If $y_{i} \geq h\left(\alpha_{j}\right)$, then the number of solutions is $\ll 1$.

Hence, if $(M(F) / m)^{5} \geq h\left(\alpha_{j}\right)$, the number of solutions is $\ll 1$ and otherwise we have $h\left(\alpha_{j}\right) \geq(M(F) / m)^{5} \gg\left(h\left(\alpha_{j}\right)^{n} / m\right)^{5}$, so $\log \log h\left(\alpha_{j}\right) \ll$ $\log \log m^{1 / n}$.

Finally we conclude that the number of primitive large solutions is $\ll$ $t\left(1+\log \log m^{1 / n}\right) \ll s\left(1+\log \log m^{1 / n}\right)$. 


\section{Bound for $\tilde{P}(F, m)$, Proof of Theorem 1.2}

8.1. Definitions of small, medium and large solutions. Let $\boldsymbol{x}=(x, y) \in$ $\mathbb{Z}^{2}$. We define

$$
|\boldsymbol{x}|=\max (|x|,|y|), \quad\langle\boldsymbol{x}\rangle=\min (|x|,|y|) .
$$

Given $F \in C(4 s-2)$, we measure the size of possible solutions $(x, y)$ of (13) by the size of $\langle\boldsymbol{x}\rangle$ and $|\boldsymbol{x}|$.

Relative to two quantities $Y_{S}, Y_{L}$, which will be defined below in (56) and (57), we call a solution $(x, y) \in \mathbb{Z}^{2}$

$$
\begin{array}{rll}
\text { small } & \text { if } & 0 \leq\langle\boldsymbol{x}\rangle \leq Y_{S}, \\
\text { medium } & \text { if } & |\boldsymbol{x}| \leq Y_{L} \text { and }\langle\boldsymbol{x}\rangle>Y_{S}, \\
\text { large } & \text { if } & |\boldsymbol{x}|>Y_{L} .
\end{array}
$$

We will split the count of possible solutions $(x, y)$ into small, medium and large solutions. We choose the constants below to be consistent with Mueller and Schmidt's work [14]. Let $H(F)$ be the height of $F$ and let $M$ be the smallest Mahler measure among the forms equivalent to $F$. Put

$$
C=R m(2 H(F) \sqrt{n(n+1)})^{n},
$$

where $R$ is defined by (34). Pick numbers $a, b$ with $0<a<b<1$ so small that

$$
\frac{\sqrt{2} \sqrt{3+a^{2}}}{1-b}<3
$$

Put

$$
\lambda=\frac{\sqrt{2\left(n+a^{2}\right)}}{1-b},
$$

so that, by (53), $n-\lambda>0$. Note that

$$
\lambda \asymp \sqrt{n}, \quad n-\lambda \asymp n-\sqrt{n} .
$$

We define

$$
A=\frac{1}{a^{2}}\left(\log M+\frac{n}{2}\right),
$$

and

$$
\begin{gathered}
Y_{S}=\left(\left(e^{6} s\right)^{n} R^{2 s} m\right)^{\frac{1}{n-2 s}}, \\
Y_{L}=(2 C)^{1 /(n-\lambda)}\left(4 e^{A}\right)^{\lambda /(n-\lambda)} .
\end{gathered}
$$

Our definitions of $Y_{S}$ and $Y_{L}$ are the same as the quantities introduced by Mueller and Schmidt in [14, eq. 2.10 and 2.9] to distinguish between small and large solutions. With these definitions we have:

Proposition 8.1 (Mueller-Schmidt). Let $F(x, y) \in \mathbb{Z}[x, y]$ be an irreducible binary form with $s+1$ nonzero coefficients and degree $n \geq 3$. For any positive integer $m$, the number of primitive large solutions of $F(x, y) \leq m$ $i s \ll s$. 
Proposition 8.2. Let $F(x, y) \in \mathbb{Z}[x, y]$ be an irreducible binary form with $s+1$ nonzero coefficients and degree $n \geq 3 s$. For any positive integer $m$, the number of primitive medium solutions of $F(x, y) \leq m$ is

$$
\ll \begin{cases}s\left(1+\frac{\log m^{1 / n}}{\log H(F)}\right) & \text { if } n \geq s^{4} \\ (s \log s)\left(1+\frac{\log m^{1 / n}}{\log H(F)}\right) & \text { if } 9 s^{2} \leq n<s^{4} \\ (s \log s)\left(1+\frac{s+\log m^{1 / n}}{\log H(F)}\right) & \text { if } n<9 s^{2} .\end{cases}
$$

Proposition 8.1 is Mueller-Schmidt's result [14, Prop. 1]. Proposition 8.2 will be proved in section 10 of this article. We generalise to arbitrary $m$ the argument in [1, Section 5]. Small solutions are more difficult to deal with; they will be counted essentially by the proposition 8.3 below that we will prove in sections 4 and 9 .

We write $\tilde{P}_{S_{x}}(F, m), \tilde{P}_{S_{y}}(F, m)$ for the number of primitive solutions of (13) with $0 \leq x \leq Y_{S}$ and $0 \leq y \leq Y_{S}$ respectively.

Proposition 8.3. Let $F(x, y) \in \mathbb{Z}[x, y]$ be an irreducible binary form that lies in $C(4 s-2)$. Let $M$ be the smallest Mahler measure among the forms equivalent to $F$, and $m$ be a positive integer such that

$$
m \leq \frac{M}{100^{n}} .
$$

Then

$$
\max \left(\tilde{P}_{S_{x}}(F, m), \tilde{P}_{S_{y}}(F, m)\right) \ll s+\log ^{3} n+\log m^{1 / n} .
$$

Next we prove Theorem 1.2 assuming Propositions 8.2 and 8.3 . Large and medium solutions are counted by Propositions 8.1 and 8.2 respectively, so we only need to count small solutions assuming Proposition 8.3. For this we refine the argument that we used for large discriminant in section [5, Note that the argument below can be used together with Proposition 8.3 to bound small solutions because the condition on $F$ in Proposition 8.3 is $F \in C(4 s-2)$; it would not be useful if we had a condition on the number of nonzero coefficients such as in Proposition 8.2.

Let $F \in \mathbb{Z}[x, y]$ be an irreducible binary form with $s+1$ nonzero coefficients and degree $n \geq 3 s$. By Lemma 2.1, $F(x, y) \in C(4 s-2)$. Let $m$ be a positive integer. Pick the smallest prime $p$ that satisfies

$$
p>10^{6} m^{\frac{2}{n}}|D(F)|^{-\frac{1}{n(n-1)}}
$$

and consider the matrices

$$
A_{0}=\left(\begin{array}{cc}
1 & 0 \\
0 & p
\end{array}\right), \quad A_{j}=\left(\begin{array}{cc}
p & j \\
0 & 1
\end{array}\right) \quad(j=1, \ldots, p) .
$$

Recall that $F_{A_{j}} \in C(4 s-2)$ for $j=0, \ldots, p$ and $\mathbb{Z}^{2}=\cup_{j=0}^{p} A_{j} \mathbb{Z}^{2}$, so that any solution $(x, y)$ of (13) gives a solution $(u, v)=A_{j}^{-1}(x, y)$ of $2^{-n} m \leq$ 
$F_{A_{j}}(u, v)<m$ for some $j=0, \ldots, p$. Moreover, when $j \geq 1$ we have $v=y$, so if $(x, y)$ satisfies $0 \leq y \leq Y_{S}$, then also $(u, v)$ satisfies $0 \leq v \leq Y_{S}$. Hence

$$
\tilde{P}_{S_{y}}(F, m) \leq \tilde{P}\left(F_{A_{0}}, m\right)+\sum_{j=1}^{p} \tilde{P}_{S_{y}}\left(F_{A_{j}}, m\right) \text {. }
$$

For $j=0, \ldots, p$, by (11) and (60),

$$
\left|D\left(F_{A_{j}}\right)\right|=p^{n(n-1)}|D(F)|>10^{6 n(n-1)} m^{2 n-2} .
$$

Then, by (8),

$$
10^{6 n(n-1)} m^{2 n-2} \leq\left|D\left(F_{A_{j}}\right)\right| \leq n^{n} M_{j}^{2 n-2},
$$

where $M_{j}$ is the smallest Mahler measure among the forms equivalent to $F_{A_{j}}$. Hence, $m \leq M_{j} / 100^{n}$, and we can apply Proposition 8.3 to $F_{A_{j}}$. We obtain that

$$
\tilde{P}_{S_{y}}\left(F_{A_{j}}, m\right) \ll s+\log ^{3} n+\log m^{1 / n} .
$$

Therefore,

$$
\begin{aligned}
\tilde{P}_{S_{y}}(F, m) & \ll \tilde{P}\left(F_{A_{0}}, m\right)+p\left(s+\log ^{3} n+\log m^{1 / n}\right) \\
& \ll \tilde{P}\left(F_{A_{0}}, m\right)+m^{2 / n}|D(F)|^{-\frac{1}{n(n-1)}}\left(s+\log ^{3} n+\log m^{1 / n}\right) .
\end{aligned}
$$

If we consider

$$
A_{0}^{\prime}=\left(\begin{array}{cc}
p & 0 \\
0 & 1
\end{array}\right), \quad A_{j}^{\prime}=\left(\begin{array}{cc}
1 & 0 \\
j & p
\end{array}\right) \quad(j=1, \ldots, p),
$$

any solution of (13) gives a solution $(u, v)$ of $2^{-n} m \leq F_{A_{j}^{\prime}}(u, v)<m$ for some $j=0, \ldots, p$. Similarly as above, when $j \geq 1, u=x$ and we conclude that

$$
\tilde{P}_{S_{x}}(F, m) \ll \tilde{P}\left(F_{A_{0}}^{\prime}, m\right)+m^{2 / n}|D(F)|^{-\frac{1}{n(n-1)}}\left(s+\log ^{3} n+\log m^{1 / n}\right) .
$$

The forms $F_{A_{0}}$ and $F_{A_{0}}^{\prime}$ have $s+1$ nonzero coefficients, so Propositions 8.2 and 8.1 apply together with Proposition 8.3 . We obtain that

$$
\max \left(\tilde{P}\left(F_{A_{0}}^{\prime}, m\right), \tilde{P}\left(F_{A_{0}}, m\right)\right) \ll c(s)\left(1+\log m^{1 / n}\right)+\log ^{3} n,
$$

where $c(s)$ is defined in (므).

Finally, the number of primitive small solutions of (13) is

$$
\ll\left(c(s)\left(1+\log m^{1 / n}\right)+\log ^{3} n\right) m^{2 / n}|D(F)|^{-\frac{1}{n(n-1)}} .
$$

\section{Proof of Proposition 8.3}

We apply the inequality (37) to $Y=Y_{S}$, defined by (56). Since $\frac{n}{n-2 s} \leq 3$,

$$
n \log Y_{S}+n \log (6 R+5) \ll \log m+n \log s+n \log ^{3} n .
$$

We also have, by (58),

$$
\log \left(M /\left(6^{n} m\right)\right) \gg n
$$


We conclude that

$$
\left|\mathfrak{X} \cup \mathfrak{X}_{0}\right| \ll \frac{\log m}{n}+\log s+\log ^{3} n .
$$

Hence

$$
\max \left(\tilde{P}_{S_{x}}(F, m), \tilde{P}_{S_{y}}(F, m)\right) \ll s+\log ^{3} n+\log m^{1 / n} .
$$

\section{Medium solutions, proof of Proposition 8.2}

Let $F(x, y) \in \mathbb{Z}[x, y]$ be a binary form of degree $n$ with $s$ non-zero coefficients. Let $m$ be a positive integer.

We divide the interval $\left[Y_{S}, Y_{L}\right]$ into $N+1$ subintervals, where $Y_{S}$ and $Y_{L}$ are defined in (56) and (57) and $N$ depends on $s$ and is defined below. We will show that there are only few solutions $(x, y)$ with $\langle\boldsymbol{x}\rangle$ in each of these subintervals. In this section we will assume $n \geq 3 s$. We define the positive integer $N=N(n, s)$ as follows.

If $n \geq s^{4}$, we put $N=2$. Otherwise, we put $k=\sqrt{n}$ if $9 s^{2} \leq n<s^{4}$ and $k=n$ if $n<9 s^{2}$, and choose $N \in \mathbb{N}$ such that

$$
3 s^{1+\frac{1}{N}} \leq k \leq 3 s^{1+\frac{1}{N-1}} .
$$

The inequality (62) leads to

$$
N \leq \frac{\log s}{\log k-\log s}
$$

For $\ell=1, \ldots, N$, we define

$$
Y_{\ell}=Y_{S} H(F) \frac{1}{s^{1-(\ell-1) / N}} .
$$

We put

$$
Y_{0}=Y_{S} \quad \text { and } \quad Y_{N+1}=Y_{L} .
$$

Proposition 10.1. There is a set $\boldsymbol{T}$ of roots of $F(x, 1)$ and a set $\boldsymbol{T}^{*}$ of roots of $F(1, y)$, both with cardinalities $\leq 6 s+4$, such that any solution $(x, y)$ of $|F(x, y)| \leq m$ with $\langle\boldsymbol{x}\rangle \geq Y_{S}$ either has

$$
\left|\alpha-\frac{x}{y}\right|<\frac{R(n s)^{2}}{H(F)^{(1 / s)-(1 / n)}}\left(\frac{\left(4 e^{3} s\right)^{n} m}{y^{n}}\right)^{1 / s}
$$

with some $\alpha \in \boldsymbol{T}$ and $R$ defined by (34), or has

$$
\left|\alpha^{*}-\frac{y}{x}\right|<\frac{R(n s)^{2}}{H(F)^{(1 / s)-(1 / n)}}\left(\frac{\left(4 e^{3} s\right)^{n} m}{x^{n}}\right)^{1 / s}
$$

for some $\alpha^{*} \in \boldsymbol{T}^{*}$.

Proof. This is Lemma 17 of [14].

Let $\alpha \in \boldsymbol{T}$. For $\ell \in\{0, \ldots, N\}$, let $\left(x_{1}, y_{1}\right), \ldots,\left(x_{w_{\ell}}, y_{w_{\ell}}\right)$ be the primitive solutions of $|F(x, y)| \leq m$, with $Y_{\ell}<y_{i} \leq Y_{\ell+1}$, satisfying (64) and ordered so that

$$
Y_{\ell}<y_{1} \leq \ldots \leq y_{w_{\ell}} \leq Y_{\ell+1}
$$


By (64), we have that

$$
\frac{1}{y_{i} y_{i+1}} \leq\left|\frac{x_{i+1}}{y_{i+1}}-\frac{x_{i}}{y_{i}}\right| \leq \frac{U}{H(F)^{\frac{1}{s}-\frac{1}{n}} y_{i}^{\frac{n}{s}}},
$$

with

$$
U=2 R(n s)^{2}\left(4 e^{3} s\right)^{n / s} m^{1 / s} .
$$

Therefore, for solutions $(x, y)$ with $y \in\left(Y_{\ell}, Y_{\ell+1}\right]$, we have

$$
y_{i+1} \geq U^{-1} H(F)^{\frac{1}{s}-\frac{1}{n}} y_{i}^{\frac{n}{s}-1} \geq U^{-1} H(F)^{\frac{1}{s}-\frac{1}{n}} Y_{\ell}^{\frac{n}{s}-2} y_{i} .
$$

First we will give an estimate for the number of primitive solutions in $\left(Y_{0}, Y_{1}\right]$. By the definition of $Y_{S}=Y_{0}$ and since $n \geq 3 s$, we have

$$
U^{-1} Y_{S}^{\frac{n}{s}-2} \geq \frac{e^{\frac{3 n}{s}} R}{2(n s)^{2} 4^{\frac{n}{s}}} \geq 1 .
$$

For $\ell=0$, we have by (66) and (67) that $y_{i+1} \geq H(F)^{\frac{1}{s}-\frac{1}{n}} y_{i}$, so $y_{w_{0}} \geq$ $\left(H(F)^{\frac{1}{s}-\frac{1}{n}}\right)^{\left(w_{0}-1\right)} y_{1}$. So we have

$$
Y_{1} \geq y_{w_{0}} \geq\left(H(F)^{\frac{1}{s}-\frac{1}{n}}\right)^{\left(w_{0}-1\right)} Y_{0}
$$

and

$$
w_{0}-1 \leq \frac{\log \frac{Y_{1}}{Y_{0}}}{\left(\frac{1}{s}-\frac{1}{n}\right) \log H(F)}<\frac{1}{1-\frac{s}{n}} \leq \frac{3}{2},
$$

since $\log \frac{Y_{1}}{Y_{0}}=\frac{1}{s} \log H(F)$ and $n \geq 3 s$.

For $1 \leq \ell<N$, by (66) and (67) we have that $y_{i+1} \geq U^{-1} H(F)^{\frac{1}{s}-\frac{1}{n}} Y_{S}^{\frac{n}{s}-2} H(F)^{\frac{n / s-2}{s^{1-(\ell-1) / N}}} y_{i} \geq H(F)^{\frac{n}{s^{2-(\ell-1) / N}}-\frac{2}{s^{1-(\ell-1) / N}}+\frac{1}{s}-\frac{1}{n}} y_{i}$.

Therefore,

$$
y_{w_{\ell}} \geq H(F)\left(\frac{n}{s^{2-(\ell-1) / N}}-\frac{2}{s^{1-(\ell-1) / N}}+\frac{1}{s}-\frac{1}{n}\right)\left(w_{\ell}-1\right) y_{1},
$$

and since $Y_{\ell}<y_{1} \leq y_{w_{\ell}} \leq Y_{\ell+1}$, we have

$$
w_{\ell}-1 \leq \frac{\log \frac{Y_{\ell+1}}{Y_{\ell}}}{\left(\frac{n}{s^{2-(\ell-1) / N}}-\frac{2}{s^{1-(\ell-1) / N}}+\frac{1}{s}-\frac{1}{n}\right) \log H(F)} .
$$

For $\ell<N$, since $\log \frac{Y_{\ell+1}}{Y_{\ell}}<\frac{1}{s^{1-\ell / N}} \log H(F)$ and $n \geq 3 s^{1+1 / N}$,

$$
w_{\ell}-1 \leq \frac{1}{\frac{n}{s^{1+1 / N}}-\frac{2}{s^{1 / N}}+\frac{1}{s^{\ell / N}}-\frac{s^{1-\ell / N}}{n}} \leq 1 .
$$

For $\ell=N$, we have

$$
\log Y_{\ell+1}=\log Y_{L} \ll \log H(F)+\log m^{1 / n}+\sqrt{n},
$$

so

$$
w_{N}-1 \ll \frac{\log H(F)+\log m^{1 / n}+\sqrt{n}}{\left(\frac{n}{s^{1+1 / N}}-\frac{2}{s^{1 / N}}+\frac{1}{s}-\frac{1}{n}\right) \log H(F)} .
$$


If $n<9 s^{2}$, then $\sqrt{n}<3 s$ and

$$
w_{N}-1 \ll 1+\frac{s+\log m^{1 / n}}{\log H(F)} .
$$

If $n \geq 9 s^{2}$, then by (62),

$$
\frac{\sqrt{n}}{\frac{n}{s^{1+1 / N}}-\frac{2}{s^{1 / N}}+\frac{1}{s}-\frac{1}{n}} \ll 1,
$$

and

$$
w_{N}-1 \ll 1+\frac{\log m^{1 / n}}{\log H(F)} .
$$

We conclude that the number of primitive medium solutions of (64) for each $\alpha \in \boldsymbol{T}$ is $\ll N+\frac{\log m^{1 / n}}{\log H(F)}$ when $n \geq 9 s^{2}$ and $\ll N+\frac{s+\log m^{1 / n}}{\log H(F)}$ when $n<9 s^{2}$. In a similar way, we obtain the same bound for the number of primitive medium solutions of (65) for each $\alpha^{*} \in \boldsymbol{T}^{*}$. Using Proposition 10.1 and the fact that $N=2$ for $n \geq s^{4}$ and $N \leq \log s$ for $n<s^{4}$, we obtain Proposition 8.2.

\section{REFERENCES}

[1] S. Akhtari and P. Bengoechea, Representation of small integers by sparse binary forms, submitted (2019).

[2] B.J. Birch and J.R. Merriman, Finiteness theorems for binary forms with given discriminant, Proc. London Math. Soc. 25 (1972), 385-394.

[3] E. Bombieri, W. M. Schmidt, On Thue's equation, Invent. Math. 88 (1987), 69-81.

[4] S. Chowla, Contributions to the analytic theory of numbers (II). J. Indian Math. Soc. 20 (1933), 120-128.

[5] Evertse, J.-H.: Upper bounds for the number of solutions of diophantine equations. Math. Centrum. Amsterdam, pp. 1-127 (1983).

[6] J. H. Evertse and K. Győry, Thue inequalities with a small number of solutions, in: The mathematical heritage of C.F. Gauss, World Scientific Publ. Co., Singapore, 1991, 204-224.

[7] K. Győry, Thue inequalities with a small number of primitive solutions, Period. Math. Hungar. 42 (2001), no. 1-2, 199-209.

[8] D. Lewis and K. Mahler, Representation of integers by binary forms, Acta Arith. 6 (1961), 333-363.

[9] K. Mahler, Zur Approximation algebraischer Zahlen III. Über die mittlere Anzahl der Darstellungen grosser Zahlen durch binäre Formen, Acta. Math. 62 (1933), 91-166.

[10] K. Mahler, On the lattice points on curves of genus 1. Proc. London Math. Soc. (2) 39 (1935), 431-466.

[11] K. Mahler, On two extremum properties of polynomials, Illinois J. Math. 7 1(963) 681-701.

[12] K. Mahler, An inequality for the discriminant of a polynomial, Michigan Math. J. 11 (1964), 257-262.

[13] J. Mueller and W. M. Schmidt, Trinomial Thue equations and inequalities, J. Reine Angew. Math., 379 (1987), 76-99.

[14] J. Mueller and W. M. Schmidt, Thue's equation and a conjecture of Siegel, Acta Math. 160 (1988), no. 3-4, 207-247.

[15] W. M. Schmidt, Thue equations with few coefficients, Trans. Amer. Math. Soc. 303 (1987), 241-255. 
[16] W.M. Schmidt, Diophantine Approximations and Diophantine equations, Lecture Notes in Mathematics, Vol. 1467, Springer-Verlag, New York, 1991.

[17] Siegel, C. L., Über einige Anwendungen diophantischer Approximationen. Abh. Preuss. Akad. Wiss. Phys.-math. Kl., 1929, Nr. 1.

[18] J. H. Silverman, Integer points on curves of genus 1. J. London Math. Soc. (2) 28 (1983), 1-7.

[19] C. L. Stewart, On the number of solutions of polynomial congruences and Thue equations, J. Amer. Math. Soc. 4 (1991), 793-835.

[20] A. Thue, Berechnung aller Lösungen gewisser Gleichungen von der form $a x^{r}-b y^{r}=f$. Vid. Skrifter I Mat.-Naturv. Klasse (1918), 1-9.

[21] J. L. Thunder, on Thue inequalities and a conjecture of Schmidt, J. Number Theory 52 (1995), 319-328.

ETH, Mathematics Dept., CH-8092, Zürich, Switzerland

E-mail address: paloma.bengoechea@math.ethz.ch 